\title{
Propriedades Psicométricas da Medida de Negociação Interpessoal do Relationship Questionnaire numa Amostra Portuguesa
}

\author{
Psychometric Properties of the Interpersonal Negotiation Measure of the Relationship \\ Questionnaire in a Portuguese Sample
}

\author{
Nádia Salgado Pereira ${ }^{12}$ e Alexandra Marques-Pinto ${ }^{2}$
}

\begin{abstract}
Resumo
Em Portugal, escasseiam estudos sobre instrumentos que avaliem competências de tomada de decisão responsável. Neste sentido, o presente estudo pretendeu analisar propriedades psicométricas da medida de Negociação Interpessoal do Relationship Questionnaire, numa amostra de 197 alunos dos $2^{\circ}$ e $3^{\circ}$ ciclos do ensino básico português. Especificamente, avaliaram-se a sua validade factorial, fidedignidade e validade discriminante. Seguidamente, analisaram-se as competências globais dos alunos avaliadas pela medida de Negociação Interpessoal e as suas diferenças em função das variáveis sociodemográficas. Os resultados revelaram um melhor ajustamento do modelo unifactorial, adequada fidedignidade e poder discriminativo da medida relativamente a outros domínios da Aprendizagem Sócio-Emocional. Os resultados indicaram ainda competências adequadas ao desenvolvimento por parte dos alunos; melhores resultados por parte das raparigas do que dos rapazes; e a inexistência de diferenças significativas relativamente à idade, ao ciclo de ensino e à nacionalidade. Estes resultados atribuem relevância ao aprofundamento da medida em estudo.
\end{abstract}

Palavras-chave: aprendizagem sócio-emocional, tomada de decisão responsável, propriedades psicométricas, negociação interpessoal, $2^{\circ}$ e $3^{\circ}$ ciclos do Ensino Básico

\begin{abstract}
In Portugal, studies on instruments that assess responsible decision-making skills are scarce. Hence, the present study set out to analyze the psychometric properties of the Interpersonal Negotiation measure of the Relationship Questionnaire, in a sample of 197 pupils from 2nd and 3rd cycles of Portuguese middle schools. Factorial validity, reliability and discriminant validity were evaluated. Pupils' global skills evaluated by the Interpersonal Negotiation measure were then analyzed, as well as the socio-demographic differences. Results revealed a better fit of the one-factor model, adequate reliability, and discriminative power of the measure regarding other Socio-Emotional Learning domains. Results also indicated adequate skills of the pupils considering their stage of development; results for girls were higher in comparison with boys; and a lack of significant differences regarding age, educational cycle and nationality. These data reinforce the need to further develop the measure under study.
\end{abstract}

Keywords: socio-emotional learning, responsible decision-making, psychometric properties, interpersonal negotiation; 2nd and 3rd cycles of Middle School

Este Estudo foi financiado por uma Bolsa de Doutoramento atribuída ao primeiro autor pela Fundação para a Ciência e Tecnologia (SFRH/BD/76257/2011).

\footnotetext{
${ }^{1}$ Instituto de Ciências Sociais, Universidade de Lisboa, Avenida Professor Aníbal de Bettencourt 9, 1600-189 Lisboa, Portugal.

${ }^{2}$ CICPSI, Faculdade de Psicologia, Universidade de Lisboa, Alameda da Universidade, 1649-013 Lisboa, Portugal. Correspondência: Nádia Salgado Pereira, Departamento de Psicologia da Educação, Faculdade de Psicologia, Universidade de Lisboa, Alameda da Universidade, 1649-013 Lisboa, Portugal. Tel.: +351 913731461. E-mail: nadia.mspereira@ gmail.com
} 


\section{Introdução}

Em Portugal, os dados preliminares do $1^{\circ}$ estudo epidemiológico sobre a prevalência de problemas psicológicos apontam para uma prevalência anual de 23\% (Direcção Geral da Saúde [DGS], 2013) e os resultados de um estudo recente (Matos, Simões, Camacho, Reis, \& Equipa Aventura Social, 2015) revelaram um decréscimo da saúde mental e física dos adolescentes. $\mathrm{O}$ envolvimento em comportamentos de risco é um dos principais factores associado à saúde mental e ao bem-estar negativo dos adolescentes, podendo conduzir a dificuldades na construção de um percurso de vida bem-sucedido (Carvalho \& Novo, 2014). Entende-se, deste modo, a necessidade de intervir precocemente na área da saúde mental e do bemestar.

Ao longo da infância e da adolescência, as tarefas de desenvolvimento implicam competências pessoais e sociais que, sendo insuficientes ou estando em falta, podem resultar em dificuldades emocionais e comportamentais (Social and Character Development Research Consortium, 2010). Neste sentido, as intervenções na área da saúde mental e do bem-estar, destinadas às crianças e aos jovens, podem contribuir determinantemente para o seu desenvolvimento saudável, com repercussões positivas na idade adulta (DGS, 2013). A maioria das intervenções centra-se, actualmente, na promoção de competências, em vez de se focar no tratamento de um problema ou perturbação específicos (Moore \& Keyes, 2003). No âmbito destas intervenções, encontram-se os programas dedicados à promoção de competências sociais e emocionais, denominados de programas de Aprendizagem Sócio-Emocional (ASE).

Estes programas envolvem a aprendizagem e o desenvolvimento de um conjunto de competências sócio-emocionais, consideradas fundamentais à compreensão e à gestão de tarefas de desenvolvimento, tais como a aprendizagem escolar, a construção de relacionamentos e a adaptação às exigências sociais (Collaborative for Academic Social and Emotional Learning [CASEL], 2015). Desde o início do século, têm vindo a ser desenvolvidos em todo o mundo diversos programas de ASE nas escolas, tendo Portugal acompanhado igualmente esta tendência (e.g., Coelho, Sousa, \& Figueira, 2014; Raimundo, Marques-Pinto, \& Lima, 2013). As competências sócio-emocionais integradas nestes programas distribuem-se por cinco principais domínios, associados a um conjunto de competências específicas (CASEL, 2015; Merrell \& Gueldner, 2010): auto-conhecimento (e. g., capacidade para compreender o que se sente em cada momento), consciência social (e. g., capacidade para compreender o que os outros estão a sentir), auto-regulação (e. g., capacidade para lidar eficazmente com as emoções), competências relacionais (e. g., capacidade para estabelecer e manter relacionamentos saudáveis e gratificantes) e tomada de decisão responsável (e. g. capacidade para tomar decisões considerando as suas diferentes consequências).

Os programas de ASE têm revelado um impacto positivo nas atitudes das crianças e dos adolescentes em relação a si próprios, aos outros e à escola, nos seus comportamentos e ainda no seu desempenho escolar (Durlak et al., 2011), realçando a importância das competências sócioemocionais no desenvolvimento. Em particular, as competências sócio-emocionais de tomada de decisão responsável, quando desenvolvidas de forma ajustada, permitem uma adequada avaliação e reflexão sobre as situações (Zins \& Elias, 2007) e associam-se à capacidade para tomar decisões, considerando as normas sociais e éticas existentes, as consequências das acções e ainda o bem-estar pessoal e dos outros (CASEL, 2015). Este tipo específico de competências assume maior importância no desenvolvimento à medida que as interacções sociais aumentam de frequência e de complexidade, quando predomina uma maior necessidade de resolver problemas sociais (Denham, Ji, \& Hamre, 2010). Neste sentido, as competências de tomada de decisão responsável conduzem a que as crianças e os adolescentes sejam mais capazes de fazer escolhas construtivas e respeitadoras sobre o comportamento pessoal e as interacções sociais (CASEL, 2015), sendo, por isso, fundamentais à eficaz resolução de conflitos sociais.

A adaptação e o desenvolvimento de programas de ASE ao contexto português implica a existência de instrumentos que avaliem as $\mathrm{N}^{\circ} 44 \cdot \operatorname{Vol} .2 \cdot 65-76 \cdot 2017$ 
competências sócio-emocionais, entre outras variáveis, os quais detenham propriedades psicométricas adequadas e se encontrem devidamente adaptados às populações alvo, permitindo, assim, determinar a eficácia destas intervenções. Um dos princípios subjacente ao desenvolvimento de intervenções de ASE consiste na avaliação do impacto destes programas nas cinco áreas centrais das competências sócioemocionais - auto-conhecimento, consciência social, auto-regulação, competências relacionais e tomada de decisão responsável (Zins \& Elias, 2007). Deste modo, diversos questionários têm sido submetidos a estudos de adaptação em amostras portuguesas, constituídas por alunos de variadas idades e anos de escolaridade, possibilitando a avaliação de algumas destas dimensões sócio-emocionais.

No entanto, faltam estudos, em Portugal, que avaliem qualidades psicométricas de instrumentos de medida no domínio da tomada de decisão responsável. Dada a importância gradual deste tipo de competências sócio-emocionais ao longo do desenvolvimento ao nível intrapessoal e interpessoal (CASEL, 2015; Denham et al., 2010), valoriza-se a necessidade de adaptar medidas ao contexto português que permitam avaliar este tipo de competências nas crianças e nos adolescentes, em variados contextos (e. g., clínico). Paralelamente, é de salientar a necessidade de proceder à avaliação da eficácia dos programas de ASE em cada um dos principais domínios das competências sócio-emocionais, através de instrumentos de medida que permitam avaliar especificamente cada um deles (Zins \& Elias, 2007). No entanto, em Portugal, as competências na área da tomada de decisão responsável têm sido, muitas vezes, avaliadas, no âmbito das intervenções de ASE, recorrendo a medidas de outras competências sócio-emocionais, assumindo-se que a tomada de decisão responsável estaria também a ser avaliada de forma implícita (e.g., Raimundo et al., 2013).

Deste modo, o presente estudo pretende dar resposta a estas necessidades avaliativas no contexto português, incidindo na avaliação de propriedades psicométricas de duas das escalas do Relationship Questionnaire (Rel-Q; Schultz, Selman, \& LaRusso, 2003), as escalas de Negociação Interpessoal, as quais avaliam um Revista Iberoamericana de Diagnóstico y Evaluación - e Avaliação Psicológica. RIDEP $\cdot$ No44 · Vol.2 · 65-76 · 2017 conjunto de competências interpessoais utilizadas na negociação de conflitos com pares e com adultos, em contextos sociais.

O Rel-Q foi desenvolvido com a finalidade de responder à necessidade de avaliação de programas de promoção de competências sociais, tendo sido criado inicialmente para avaliar um programa escolar multicultural de promoção da literacia, de educação do carácter e de prevenção da violência (Schultz, Barr, \& Selman, 2001). O questionário Rel-Q permite avaliar competências associadas ao conceito de maturidade psicossocial, em crianças e em adolescentes, existindo uma versão para crianças desde a préprimária ao $4^{\circ}$ ano (Shultz \& Selman, 2004) e outra versão destinada a crianças e adolescentes, do $4^{\circ}$ ano até ao $12^{\circ}$ ano de escolaridade (Schultz, Selman, \& LaRusso, 2003). A maturidade psicossocial refere-se a um constructo desenvolvimentista envolvendo a capacidade sócio-cognitiva para diferenciar e coordenar as perspectivas sociais, considerando o próprio e os outros (LaRusso \& Selman, 2003). Segundo esta abordagem, a capacidade de coordenação das perspectivas sociais contribui para estruturar as competências psicossociais, e distribui-se num contínuo, evoluindo ao longo de diferentes níveis de desenvolvimento: nível 0 (egocentrismo), entre os 3 e os 5 anos de idade; nível 1 (unidirecionalidade), entre os 6 e os 7 anos; nível 2 (reciprocidade), entre os 7 e os 8 anos; nível 3 (mutualismo), entre os 12 e os 14 anos; e nível 4 (interdependência), entre os 15 e os 18 anos.

As escalas de Negociação Interpessoal do questionário Rel-Q, contempladas no presente estudo, medem competências interpessoais relacionadas com a negociação de conflitos sociais, enquadrando-se, assim, no âmbito da tomada de decisão responsável das competências sócio-emocionais. Estas escalas do Rel-Q encontram-se incluídas num compêndio de medidas da eficácia dos programas de ASE, dirigidas a alunos do pré-escolar ao ensino básico, utilizadas por investigadores, psicólogos, educadores e assistentes sociais, sendo identificadas, pelos autores, como medidas de avaliação do domínio da tomada de decisão responsável (Denham et al., 2010). Existem outras medidas das competências de tomada de decisão responsável, nomeadamente os questionários 
Challenging Situations Task (CST; Denham, Way, Kalb, Warren-Khot, \& Bassett, 2013) e Devereux Student Strengths Assessment (DESSA; LeBuffe, Shapiro, \& Naglieri, 2009). No entanto, desconhecem-se estudos de adaptação destes instrumentos com crianças e adolescentes, em Portugal. A opção pelas escalas de Negociação Interpessoal do Rel-Q realizou-se com base na qualidade das propriedades psicométricas das escalas, no número de itens e ainda na faixa etária da população alvo, em comparação com as medidas alternativas consideradas.

O presente estudo incide na segunda versão do Rel-Q, destinada a crianças e adolescentes do $4^{\circ}$ ano ao $12^{\circ}$ ano de escolaridade (Schultz, Selman, \& LaRusso, 2003), e constitui uma primeira abordagem à avaliação de propriedades psicométricas das duas escalas de Negociação Interpessoal do questionário, numa amostra de alunos dos $2^{\circ}$ e $3^{\circ}$ ciclos do ensino básico português. Até ao momento, desconhece-se a existência de instrumentos de medida construídos ou adaptados para o contexto português que avaliem competências sócio-emocionais neste domínio, em crianças e em adolescentes situados nesta fase de desenvolvimento. Adicionalmente, o presente estudo pretende compreender em que medida estas escalas se diferenciam de outros instrumentos de avaliação de domínios distintos da ASE.

Desta forma, o presente estudo procurou, em primeiro lugar, avaliar a validade factorial das duas escalas de Negociação Interpessoal do Rel$\mathrm{Q}$, bem como a sua fidedignidade e ainda a validade discriminante relativamente a outras medidas das demais competências de ASE, numa amostra de alunos dos $2^{\circ}$ e $3^{\circ}$ ciclos do ensino básico português. Seguidamente, as competências medidas pelas escalas foram avaliadas na globalidade dos alunos e foram ainda testadas as diferenças de médias entre os alunos em função de um conjunto de variáveis socio-demográficas.

\section{Método}

\section{Participantes}

A amostra incluiu 197 alunos dos $2^{\circ}$ e $3^{\circ}$ ciclos do ensino básico de três escolas públicas portuguesas do distrito de Lisboa, cujo nível socio-económico das populações abrangidas era médio e médio baixo. A média de idades dos participantes foi de 11 anos $(\mathrm{M}=11.37, \mathrm{DP}=1.49)$; $30 \%$ eram rapazes e $70 \%$ raparigas; $48 \%$ dos alunos frequentava o $5^{\circ}$ ano de escolaridade, $29 \%$ o $6^{\circ}$ ano, $19 \%$ o $7^{\circ}$ ano e $3 \%$ o $8^{\circ}$ ano; e $57 \%$ dos alunos tinha nacionalidade portuguesa e/ou com pais com nacionalidade portuguesa e $43 \%$ dos alunos outra nacionalidade e/ou pais com outra nacionalidade (e. g. brasileira, cabo-verdiana, guineense). Desta amostra, todos os alunos preencheram as medidas de auto-relato e 149 alunos foram ainda avaliados por uma escala preenchida pelos diretores de turma. Uma subamostra, constituída por 98 alunos, respondeu uma segunda vez às escalas de Negociação Interpessoal em estudo, oito meses após a primeira avaliação. Nesta sub-amostra a média de idades foi de 11 anos ( $M=10.7, \mathrm{DP}=93) ; 74 \%$ eram raparigas e $24 \%$ rapazes; $59 \%$ dos alunos frequentava o $5^{\circ}$ ano, $31 \%$ o $6^{\circ}$ ano, $7 \%$ o $7^{\circ}$ ano e $1 \%$ o $8^{\circ}$ ano; $61 \%$ dos alunos eram portugueses e/ou com pais com nacionalidade portuguesa e $37 \%$ dos alunos tinha outra nacionalidade e/ou os pais eram de outra nacionalidade.

\section{Medidas}

O Relationship Questionnaire (Rel-Q; Schultz, Selman, \& LaRusso, 2003), versão para crianças e adolescentes do $4^{\circ}$ ano ao $12^{\circ}$ ano de escolaridade, consiste num instrumento de medida da maturidade psicossocial. O Rel-Q é aplicado habitualmente em grupo, mas pode ser também aplicado individualmente, e consiste em 25 itens distribuídos por cinco escalas: escala da Compreensão dos Relacionamentos Interpessoais (6 itens), escala da Tomada de Perspectiva (4 itens), escala da Negociação Interpessoal Hipotética (4 itens), escala da Negociação Interpessoal Real (4 itens) e escala da Consciência do Significado Pessoal (6 itens). Cada item apresenta um dilema ou situação social com pares ou com adultos e possui quatro respostas de escolha múltipla, as quais representam pontos no contínuo de quatro dos níveis teóricos de coordenação das perspectivas sociais, segundo uma abordagem desenvolvimentista, indo do nível 0 (egocentrismo) ao nível 3 (mutualismo). O presente estudo incidiu na avaliação de 
propriedades psicométricas das duas escalas de Negociação Interpessoal, as quais têm sido consideradas medidas de avaliação no domínio da tomada de decisão responsável (Denham, Ji, \& Hamre, 2010). Cada uma destas escalas é constituída por 4 itens, as quais avaliam competências interpessoais referentes a estratégias de negociação de conflitos com os outros: a escala de Negociação Interpessoal Hipotética (e.g., "O Diretor da escola anunciou que este ano não há dinheiro para actividades desportivas e artísticas depois das aulas. Como a maioria dos alunos da escola ficaram muito tristes, a Catarina e outros alunos organizaram-se para decidir o que podem fazer para resolver o problema. A Catarina e os amigos podiam: a. Iniciar uma campanha para que todos os pais percebessem que o desporto e as artes são muito importantes para os alunos; b. Oferecerem-se para pintar o edifício da escola em troca do dinheiro para as actividades; c. Deixar de fazer as suas tarefas na escola; d. Ir à próxima reunião da direção da escola e dizer para arranjarem dinheiro para as actividades desportivas e artística") e a escala de Negociação Interpessoal Real (e.g., "Quando eu e o meu melhor amigo ou amiga não concordamos com o que vamos fazer, provavelmente eu: a. Tento convencer o meu amigo ou amiga; b. Ouço o que o meu amigo ou amiga tem a dizer e chegamos a um acordo; c. Irrito-me e vou embora sozinho(a); d. Aceito o que o meu amigo ou amiga queira fazer"). A fiabilidade relativamente baixa destas escalas ( $\alpha$ de Cronbach=.61) é associada pelos autores à natureza contextual das competências avaliadas, as quais variam, por exemplo, em função das situações apresentadas serem com pares ou com adultos (Schultz, Selman, \& LaRusso, 2003). Os respondentes avaliaram cada resposta de escolha múltipla numa escala de quatro pontos de tipo Likert (fraca, ok, boa ou excelente) e escolheram ainda qual das quatro consideravam ser a melhor resposta. Deste modo, obtiveram-se dois resultados para cada item, um resultado de avaliação da resposta e um resultado de melhor resposta. Estes resultados podem ser combinados numa média compósita da escala e, no caso de se utilizarem todas as escalas do Rel$\mathrm{Q}$, num resultado compósito das cinco escalas, o qual permite medir a maturidade psicossocial global. Revista Iberoamericana de Diagnóstico y Evaluación - e Avaliação Psicológica. RIDEP · No44 · Vol.2 · 65-76 · 2017
O Questionário de Competência Emocional (QCE; Taksic, 2000), versão portuguesa de Faria e Lima-Santos (2001), foi utilizado no presente estudo como medida de avaliação de outros domínios da ASE, distintos da tomada de decisão responsável, e consiste numa medida de autorelato cujos itens devem ser avaliados numa escala de tipo Likert que varia entre 1 (nunca) e 5 (frequentemente). O domínio do conhecimento emocional da ASE foi avaliado através da escala de Percepção Emocional do QCE, a qual mede a capacidade para perceber e compreender as emoções (15 itens, e. g. "Quando alguma coisa me desagrada, demonstro-o logo"; $\alpha$ de Cronbach $=.84$ no estudo de adaptação português e $\alpha=.86$ na presente amostra). O domínio da ASE referente à consciência social foi avaliado através da escala de Expressão Emocional do QCE, a qual avalia a capacidade para expressar e identificar as emoções (14 itens, e. g. "Percebo quando alguém tenta esconder o seu mau humor"; $\alpha$ de Cronbach=.83 no estudo de adaptação português e $\alpha=.84$ na presente amostra). O domínio da autoregulação da ASE foi avaliado através da escala da Capacidade para Lidar com a Emoção do QCE, a qual mede a capacidade para gerir e regular as emoções (16 itens, e. g. "Quando alguém me elogia, trabalho com maior entusiasmo"; $\alpha$ de Cronbach=.64 no estudo de adaptação português e $\alpha=.75$ na presente amostra).

A sub-escala Relacionamento com os Pares da Escala A do questionário School Social Behavior Scales (SSBS-2; Merrell, 2002), versão portuguesa adaptada por Raimundo et al. (2012), foi utilizada para medir o domínio dos relacionamentos da ASE. O SSBS-2 é um questionário preenchido pelos professores, utilizando uma escala do tipo Likert que varia entre 1 (nunca) e 5 (muito frequentemente). A escala de Relacionamento com os Pares mede a capacidade para estabelecer e manter relacionamentos positivos e obter aceitação social (14 itens, e. g. "Compreende os problemas e necessidades dos seus pares"; $\alpha$ de Cronbach $=.91$ no estudo de adaptação português e $\alpha=.92$ na presente amostra).

\section{Procedimento}

O presente estudo foi aprovado pelo Conselho Científico e Ético da Faculdade de Psicologia da 
Universidade de Lisboa e foi realizado após ter sido concedida autorização por parte dos conselhos administrativos das escolas envolvidas e de um consentimento informado ter sido assinado pelos encarregados de educação dos alunos participantes.

Relativamente à adaptação das duas escalas de Negociação Interpessoal do Rel-Q, primeiramente, foi realizada a tradução da versão original em língua inglesa para português, tendose utilizado também a versão em espanhol como referente. A tradução foi efetuada de forma independente por dois tradutores com formação em Psicologia que, posteriormente, confrontaram as duas traduções e realizaram os ajustes necessários para se chegar a uma tradução final das escalas. Posteriormente, um painel de juízes avaliou a qualidade da tradução das escalas. Por fim, realizou-se ainda uma retroversão da tradução, recorrendo a um tradutor bilingue, tendo-se verificado a adequação da tradução final.

Os questionários foram preenchidos no início do ano lectivo pelos alunos, em formato papel e lápis, em grupos e em salas de aula das escolas, com a presença do investigador responsável pela recolha dos dados, o qual leu instruções estandardizadas aos alunos e assegurou a confidencialidade e o anonimato das suas respostas. De forma a garantir que os alunos compreendiam o que lhes era pedido, todos os itens foram lidos em voz alta pelo investigador, à medida que os alunos respondiam. Os dados socio-demográficos foram recolhidos no início do preenchimento do protocolo de avaliação, tendo sido preenchidos pelos alunos numa folha destinada ao efeito.

\section{Análise dos dados}

A validade factorial do modelo dos autores na amostra em estudo foi avaliada através de uma Análise Factorial Confirmatória (AFC), com recurso ao software Amos SPSS (versão 22). Inicialmente, procedeu-se à análise dos itens, através da avaliação da sensibilidade da medida, com base nos valores de assimetria e de curtose, considerando-se que valores de assimetria superiores a três e valores de curtose superiores a sete representariam desvios significativos à normalidade (Maroco, 2014). O modelo foi ajustado apenas quando necessário, com base em Revista Iberoamericana de Diagnóstico y Evaluación - e Avaliação Psicológica. RIDEP · N44 · Vol.2 · 65-76 2017 fundamentação teórica e em pressupostos estatísticos, quando o valor dos índices de modificação se situou acima de 11 , sendo alteradas trajectórias e/ou eliminados itens com pesos factoriais estandardizados com valor inferior a 0.50 (Hair et al., 2006; Maroco, 2014). O modelo foi considerado ajustado aos dados com valores de CFI, TLI e GFI superiores a 0.90; o valor de RMSEA inferior a 0.06; os valores de $\chi 2 /$ df inferiores a 2 ; e os índices AIC e ECVI foram utilizados para comparar os modelos, sendo o modelo com os valores mais baixos aquele que indica melhor ajustamento (Arbuckle, 2008; Browne \& Cudeck, 1992; Hu \& Bentler, 1999; Maroco, 2014). A fidedignidade foi avaliada de duas formas, através do valor dos alfas de Cronbach para avaliar a consistência interna das escalas e através do valor do coeficiente de correlação de Pearson de forma a avaliar a fidedignidade do teste - re-teste na subamostra $(\mathrm{N}=98)$. Apesar de habitualmente se considerar que os valores que indicam boa consistência interna se situam acima de .7, os valores acima de .6 são igualmente considerados adequados para investigações exploratórias (Nunnally, 1978), como é o caso do presente estudo. A validade discriminante foi avaliada com base nos resultados das correlações de Pearson entre as escalas de Negociação Interpessoal do Rel-Q e as escalas do QCE e do SSBS-2.

Realizaram-se análises descritivas das variáveis em estudo, recorrendo à média e ao desvio-padrão. Efectuaram-se ainda testes T, com base no modelo factorial das escalas de Negociação Interpessoal do Rel-Q ajustado à amostra, de forma a testar diferenças de médias em função das variáveis socio-demográficas relativas ao género, idade, ciclo de ensino e nacionalidade. Segundo a abordagem conceptual dos autores do Rel-Q, ocorre um aumento significativo das competências de maturidade psicossocial ao longo do desenvolvimento, a cada intervalo de 4 anos, sendo um desses progressos observado no $6^{\circ}$ ano, por volta dos 11 anos de idade (Schultz, Selman, \& LaRusso, 2003). Deste modo, na comparação dos resultados relativamente à idade consideraram-se dois grupos de idades diferentes, um grupo com os alunos de idade inferior a 11 anos e um grupo com os alunos de idade igual ou superior a 11 anos. 


\section{Resultados}

\section{Validade factorial e fidedignidade}

A avaliação da sensibilidade da medida revelou uma adequada distribuição dos resultados, verificando-se que todos os itens apresentaram valores de assimetria e de curtose próximos da distribuição normal. Na AFC realizada com a finalidade de testar o modelo de medida original proposto pelos autores (bifactorial, com 4 itens em cada escala), os valores de alguns dos índices de qualidade do ajustamento considerados indicaram um ajustamento sofrível do modelo aos dados da presente amostra $(\chi 2 / \mathrm{df}=1.91, \mathrm{CFI}=.90, \mathrm{GFI}=.96$, $\mathrm{TLI}=.85$, RMSEA=.07). Os valores dos índices AIC e ECVI foram respectivamente de 70.25 e .36 e os valores dos índices de modificação situaramse todos abaixo de 11. De forma a melhorar o ajustamento, optou-se por retirar alternadamente os itens com pesos factoriais estandardizados mais baixos (.18 no item 8 e 0.30 no item 5) e o modelo sem o item 5 revelou um bom ajustamento à amostra $(\chi 2 / \mathrm{df}=1.41, \mathrm{CFI}=.96, \mathrm{GFI}=.97$, TLI=.94, RMSEA=.05). Os valores dos índices de modificação situaram-se todos abaixo de 11 e os valores dos índices AIC e ECVI baixaram para 48.32 e 0.25 respectivamente, indicando um melhor ajustamento deste modelo. No entanto, a avaliação da consistência interna das escalas revelou valores dos alfas de Cronbach inaceitáveis, tanto na escala de Negociação Interpessoal Hipotética $(\alpha=.55)$ como na escala de Negociação Interpessoal Real $(\alpha=.45)$. Deste modo, optou-se por testar um modelo unifactorial alternativo, agrupando os 8 itens das duas escalas originais numa escala única, indo ao encontro de um outro estudo que recorreu igualmente a uma estrutura unifactorial (Nakkula \& Nikitopoulos, 2001). Os resultados da AFC revelaram um ajustamento sofrível, em alguns dos índices de ajustamento utilizados, do modelo unifactorial constituído pelos 8 itens $(\chi 2 / \mathrm{df}=1.82, \mathrm{CFI}=.90$, GFI=.96, TLI=.86, RMSEA=.06), de forma idêntica ao ajustamento do modelo original com quatro itens em cada uma das duas escalas. Os valores dos índices AIC e ECVI foram, contudo, menores do que os do modelo original, respectivamente de 68.51 e .35 . De modo a melhorar o ajustamento, optou-se por retirar, alternadamente, os itens com pesos factoriais estandardizados mais baixos (.19 no item 8 e .31 no item 5) e testar o ajustamento do modelo. Verificou-se que, retirando o item 5, apesar de o item 8 assumir o peso factorial estandardizado mais baixo dos dois itens, o modelo apresentou um bom ajustamento à amostra $(\chi 2 / \mathrm{df}=1.44$, CFI=.96, GFI=.97, TLI=.94, RMSEA=.05) $\mathrm{e}$ menores índices de AIC e de ECVI, respectivamente de 48.15 e 0.25 . O alfa de Cronbach deste modelo foi de 0.66, revelando uma adequada consistência interna, considerando a natureza exploratória do presente estudo. Deste modo, todos os índices de ajustamento revelaram que o modelo unifactorial, composto por 7 itens (sem o item 5), foi o modelo com maior adequação à amostra do presente estudo, tal como se encontra representado na Figura 1.

Os resultados da análise teste-re-teste realizada relativamente à sub-amostra $(\mathrm{N}=98)$ indicaram que a correlação entre o teste $(\mathrm{M}=2.33$; $\mathrm{DP}=.30)$ e o re-teste $(\mathrm{M}=2.34 ; \quad \mathrm{DP}=.31)$ foi altamente significativa, positiva e moderada $(r=.421, p=0)$.

$X 2(14)=20,146 ; p=, 126 ; X 2 / g \mid=1,439 ;$ $\mathrm{CFI}=$ 958; $\mathrm{PCFI}=639 ; \mathrm{GFI}=972 \cdot \mathrm{TL}, 938 ; \mathrm{RMSEA}=047 ; \mathrm{P}(\mathrm{msea} \mathrm{D}=0.05)=494$

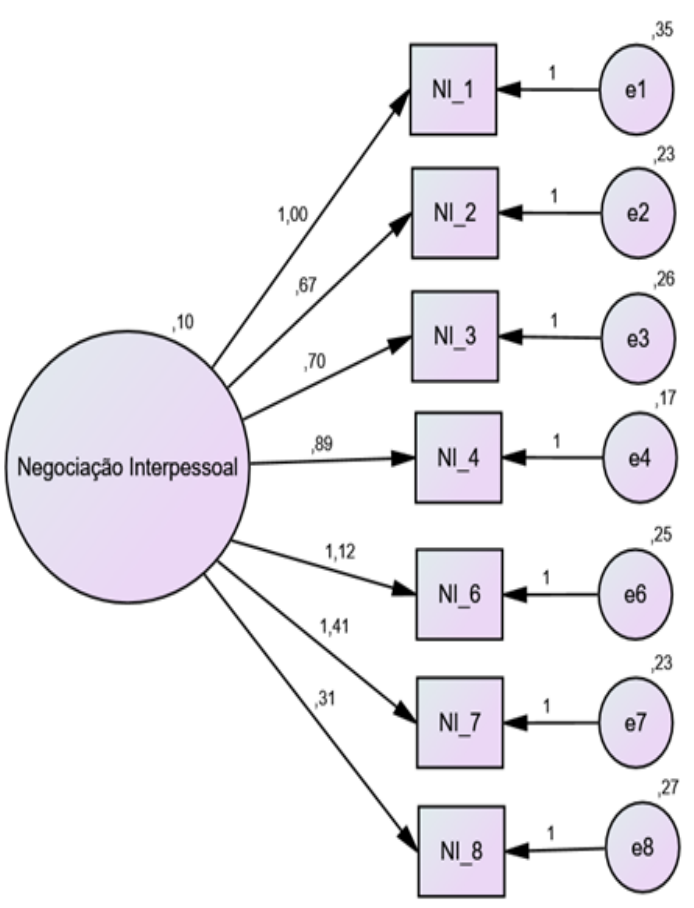

Figura 1. Análise Factorial Confirmatória da medida de Negociação Interpessoal do Rel-Q 


\section{Validade discriminante}

A validade discriminante das escalas em estudo foi avaliada com base nos resultados apresentados no Quadro 1, referentes às correlações entre a média da medida de Negociação Interpessoal do Rel-Q e as médias das três escalas do QCE (Percepção Emocional, Expressão Emocional e Capacidade para Lidar com a Emoção), bem como a médias da subescala Relacionamento com os Pares do questionário SSBS-2. Os resultados indicaram correlações significativas positivas e baixas entre as escalas do Rel-Q e as escalas de Percepção Emocional ( $r=.151, p=.03)$ e da Capacidade para Lidar com a Emoção $(r=.204, p=.004)$ do QCE. Relativamente às escalas de Expressão Emocional e de Relacionamento com os Pares, não foram encontradas correlações significativas.

Quadro 1. Média, desvio-padrão e resultados das correlações de Pearson entre a medida de

Negociação Interpessoal do Rel-Q e as restantes escalas em estudo

\begin{tabular}{lcccc}
\hline Variáveis & $M$ & $S D$ & $r$ & $\mathrm{p}$ \\
\hline Escala de Percepção & 4.55 & .85 & $.151^{*}$ & .03 \\
$\begin{array}{l}\text { Emocional do QCE } \\
\text { Escala de Expressão }\end{array}$ & 4.52 & .82 & .087 & .23 \\
$\begin{array}{l}\text { Emocional do QCE } \\
\text { Escala da Capacidade }\end{array}$ & & & & \\
para Lidar com a & 4.63 & .72 & $.204^{* *}$ & .004 \\
$\begin{array}{l}\text { Emoção do QCE } \\
\text { Escala do }\end{array}$ & & & & \\
$\begin{array}{l}\text { Relacionamento com } \\
\text { os Pares do SSBS-2 }\end{array}$ & 3.38 & .77 & .007 & .931 \\
\hline
\end{tabular}

Nota. $* p<.05, * * p<.01$

\section{Caracterização das competências interpessoais dos alunos}

A avaliação das competências na medida de Negociação Interpessoal do Rel-Q (modelo unifactorial) encontra-se representada no Quadro 2. Os resultados globais indicaram uma média de $2.28(\mathrm{SD}=.34)$. Os resultados dos testes $\mathrm{T}$ indicaram que a média das raparigas $(\mathrm{M}=2.33$, $\mathrm{SD}=.33$ ) foi significativamente superior à média dos rapazes $(\mathrm{M}=2.17, \mathrm{SD}=.33), \mathrm{t}(192)=-3.097$, $p=.002$. Relativamente às restantes variáveis socio-demográficas referentes à idade (comparação entre os alunos com idade inferior a 11 anos e idade igual ou superior a 11 anos), ciclo de ensino e nacionalidade, não foram encontradas
Quadro 2. Resultados dos testes T relativamente às diferenças entre os alunos considerando as variáveis socio-demográficas género, idade, ciclo de ensino e nacionalidade

\begin{tabular}{lcccccc}
\hline & $\mathrm{N}$ & $\mathrm{M}$ & $\mathrm{DP}$ & $\mathrm{t}$ & $\mathrm{df}$ & $\mathrm{p}$ \\
\hline Género & & & & & & \\
Raparigas & 135 & 2.33 & .33 & & & \\
Rapazes & 59 & 2.17 & .33 & & & \\
Idade & & & & & & \\
$<11$ anos & 121 & 2.28 & .33 & & & \\
$\geq 11$ anos & 73 & 2.28 & .35 & .023 & 192 & .98 \\
Ciclo de ensino & & & & & & \\
$2^{\circ}$ ciclo & 152 & 2.28 & .32 & -.208 & 192 & .84 \\
$3^{\circ}$ ciclo & 42 & 2.29 & .41 & & & \\
Nacionalidade & & & & & & \\
Portuguesa & 112 & 2.32 & .33 & 1.78 & 192 & 0.08 \\
Outra & 80 & 2.24 & .33 & & & \\
\hline
\end{tabular}

diferenças estatisticamente significativas entre os alunos.

\section{Discussão}

A presente investigação teve a finalidade de dar resposta à falta de estudos sobre instrumentos de avaliação no domínio da tomada de decisão responsável das competências sócio-emocionais, no âmbito das intervenções de Aprendizagem Sócio-Emocional (ASE). Neste sentido, o presente estudo pretendeu avaliar propriedades psicométricas das escalas de Negociação Interpessoal do Rel-Q, numa amostra de alunos $\operatorname{dos} 2^{\circ}$ e $3^{\circ}$ ciclos do ensino básico português, designadamente a sua validade factorial, fidedignidade e validade discriminante relativamente a outras medidas de domínios distintos da ASE. O presente estudo pretendeu ainda avaliar as competências medidas por essas escalas na globalidade dos alunos e em função das suas diferenças socio-demográficas.

Os resultados relativos à validade factorial das escalas indicaram que uma estrutura unifactorial, constituída por sete itens, obteve um melhor ajustamento na amostra do presente estudo do que o modelo bifactorial original proposto pelos autores do Rel-Q (Schultz, Selman, \& LaRusso, 2003), constituído por quatro itens em cada 
escala, tal como já tinha sido observado no estudo de Nakkula e Nikitopoulos (2001).

Os resultados indicaram ainda uma fidedignidade aceitável para esta solução factorial. Apesar do valor do alfa de Cronbach encontrado ter sido inferior a $.70(\alpha=.66)$, adequa-se ao carácter exploratório da presente investigação (Nunnally, 1978), assim como à natureza contextual do tipo de competências avaliadas pelas escalas (Schultz, Selman, \& LaRusso, 2003). Paralelamente, os resultados da análise teste-re-teste realizada indicaram a existência de uma correlação significativa, moderada e positiva, apontando também para a fidedignidade das escalas. O facto de a correlação encontrada ter sido moderada pode associar-se à natureza subjectiva, temporal e contextual das medidas de auto-relato, as quais dependem igualmente das capacidades cognitivas, linguísticas e de leitura dos respondentes (Elliott, Frey, \& Davies, 2015) e podem ainda conduzir a enviesamentos nas respostas em função de diversos factores como, por exemplo, a desejabilidade social (Espinosa, Menotti, \& Reyes-Lagunes, 2008).

Relativamente à validade discriminante, a medida de Negociação Interpessoal do Rel-Q apresentou correlações positivas e fracas com as escalas de Percepção Emocional (domínio da consciência social) e da Capacidade para Lidar com a Emoção (domínio da auto-regulação) tendo havido uma ausência de correlação com as escalas de Expressão Emocional (domínio do conhecimento emocional) e de Relacionamento com os Pares (domínio dos relacionamentos). Estes resultados indicaram que as competências avaliadas pela medida de Negociação Interpessoal do Rel-Q se diferenciam das competências avaliadas pelas restantes escalas, conferindo-lhe, assim, poder discriminante.

Deste modo, a medida de Negociação Interpessoal constitui uma adequada forma de avaliação do domínio da tomada de decisão responsável, de acordo com os resultados do presente estudo, os quais reforçam igualmente a ideia de este ser um constructo teórico diferenciado dos restantes domínios da ASE (CASEL, 2015).

Os resultados referentes às competências avaliadas pela medida de Negociação Interpessoal do Rel-Q, segundo a abordagem conceptual do Revista Iberoamericana de Diagnóstico y Evaluación - e Avaliação Psicológica. RIDEP · No44 · Vol.2 · 65-76 2017 questionário (LaRusso \& Selman, 2003), indicaram que a média dos alunos $(\mathrm{M}=2.28$; $\mathrm{SD}=.33$ ) se situou no nível 2 de desenvolvimento (reciprocidade), no qual as estratégias interpessoais de negociação se baseiam na cooperação e surgem habitualmente entre os 7 e os 8 anos de idade. Como a amostra do presente estudo envolveu simultaneamente alunos dos $2^{\circ} \mathrm{e}$ $3^{\circ}$ ciclos do ensino básico, com diferentes idades compreendidas entre os 9 e os 16 anos, e, como os dados do Rel-Q indicam que existe habitualmente um aumento significativo nas competências avaliadas a partir dos 11 anos (Schultz, Selman, \& LaRusso, 2003), fez-se uma comparação entre os resultados obtidos pelos alunos com idade inferior a 11 anos e os resultados dos alunos com idade igual ou superior a 11 anos. Estes resultados indicaram a inexistência de diferenças significativas entre ambos os grupos, o que sugere que o segundo grupo de alunos possa manifestar dificuldades ao nível das competências interpessoais de negociação, uma vez que seria esperado um aumento significativo da média deste grupo, em comparação com o grupo de alunos mais novos. Em concordância com os resultados relativos à idade, também não foram encontradas diferenças entre os alunos consoante o seu ciclo de ensino. Estes dados conferem pertinência à necessidade de desenvolver programas de ASE, no ensino básico português, que promovam competências no domínio da tomada de decisão responsável, tais como a utilização de estratégias de resolução de problemas eficazes, a capacidade de avaliar e de reflectir sobre as diferentes alternativas existentes na vida e desenvolver um sentido de responsabilidade pessoal, moral e ético, consideradas essenciais à saúde mental e ao bemestar positivos (Merrell \& Gueldner, 2010).

Quanto às diferenças de género encontradas, tendo as raparigas evidenciado melhores resultados do que os rapazes, este resultado vai ao encontro de estudos do Rel-Q com outras amostras (Adalbjarnardettir, 2002; Schultz, Selman, \& LaRusso, 2003) e parece sugerir diferenças de género ao nível da socialização no que se refere às competências de tomada de decisão responsável. De acordo com estudos que revelaram que as raparigas são mais encorajadas a lidar com as emoções do que os rapazes (Baillargeon et al., 2007; Maguire, Niens, 
McCann, \& Connolly, 2015; Steinberg, 2008), as diferenças de género na socialização poderão, assim, contribuir para que as raparigas desenvolvam melhores competências sócioemocionais do que os rapazes.

A ausência de diferenças em função da nacionalidade dos alunos parece sugerir que as competências avaliadas pela medida de Negociação Interpessoal do Rel-Q não variam em função de diferenças culturais observadas no domínio da tomada de decisão responsável, como, por exemplo, os indivíduos pertencentes a culturas mais individualistas tenderem a tomar decisões com base em necessidades pessoais e os indivíduos de culturas essencialmente colectivistas contemplarem mais os interesses do grupo quando tomam decisões (Hecht \& Shin, 2015).

O presente estudo revela um conjunto de limitações que é importante assinalar. Em primeiro lugar, a amostra em estudo envolveu um maior número de raparigas, um número mais elevado de alunos do $2^{\circ}$ ciclo com 10 e 11 anos e ainda um maior número de alunos com nacionalidade portuguesa. Neste sentido, os resultados anteriores devem ser utilizados cuidadosamente, considerando a heterogeneidade existente na amostra em relação às variáveis socio-demográficas. Além disso, a amostra do presente estudo foi recolhida por conveniência, não sendo, como tal, necessariamente representativa da população portuguesa. Outras limitações deste estudo referem-se ao menor tamanho da amostra do SSBS-2 em comparação com a amostra total e ao facto de cada professor ter avaliado vários alunos, o que poderá ter conduzido a alguns enviesamentos nos resultados. Outra limitação ainda diz respeito ao valor moderado da correlação encontrado na análise referente ao teste-re-teste, já anteriormente referido.

Apesar das limitações, do presente estudo decorre um conjunto de importantes implicações, tanto para a investigação como para a prática. $\mathrm{O}$ maior contributo consiste na possibilidade de dar resposta à falta de estudos sobre instrumentos de avaliação das competências sócio-emocionais no domínio da tomada de decisão responsável, em Portugal. Esta é uma necessidade sentida, em particular, no contexto dos programas de ASE Revista Iberoamericana de Diagnóstico y Evaluación - e Avaliação Psicológica. RIDEP · №44 · Vol.2 · 65-76 · 2017 desenvolvidos e adaptados ao contexto português. Neste sentido, o presente estudo atribui relevo ao potencial das escalas de Negociação Interpessoal do Rel-Q enquanto medida de avaliação de competências no domínio da tomada de decisão responsável da ASE, em alunos dos $2^{\circ}$ e $3^{\circ}$ ciclos do ensino básico português. Paralelamente, os resultados do presente estudo contribuem também para reforçar a ideia pré-existente de que o domínio da tomada de decisão responsável constitui um constructo teórico diferenciado de outros domínios das competências sócioemocionais (CASEL, 2015) e que, como tal, deve ser avaliado de forma específica em vez de se assumir que tal competência será implicitamente avaliada por medidas de avaliação das demais competências de ASE.

Deste modo, os resultados da presente investigação foram promissores, incentivando ao aprofundamento do estudo da medida de Negociação Interpessoal do Rel-Q. Seria importante realizar estudos futuros sobre a análise das qualidades psicométricas desta medida com amostras mais alargadas da população portuguesa, incluindo igualmente a avaliação de outros tipos de validade, tais como a validade convergente e de critério. Além disso, seria igualmente importante realizar de futuro estudos sobre a invariância da medida relativamente a variáveis socio-demográficas como o género, a idade, o ciclo de ensino e a nacionalidade, de forma a conduzir a uma melhor compreensão das suas forças e limitações. Por seu lado, estudos futuros, centrados em medidas de avaliação das competências sócio-emocionais, poderiam ainda centrar-se na melhor definição, no contexto português, dos domínios da ASE que cada instrumento já existente e adaptado permite efetivamente avaliar. Este tipo de estudos contribuiria para consolidar conhecimentos científicos no âmbito da avaliação da eficácia de intervenções de ASE em Portugal, com importantes implicações igualmente para a prática.

\section{Referencias}

Adalbjarnardottir, S. (2002). Adolescent psychosocial maturity and alcohol use: 
Quantitative and qualitative analysis of longitudinal data. Adolescence, 37(145), 1953.

Arbuckle, J. L. (2008). Amos 17 user's guide. Chigago, IL: SPSS.

Baillargeon, R. H., Zoccolillo, M., Keenan, K., Côté, S., Pérusse, D., Wu, H. X., Boivin, M., \& Tremblay, R. E. (2007). Gender differences in physical aggression: A prospective population-based survey of children before and after 2 years of age. Developmental Psychology, 43, 13-26. doi: 10.1037/00121649.43.1.13

Browne, M. W., \& Cudeck, R. (1992). Alternative ways of assessing model fit. Sociological Methods and Research, 21, 230-258. doi: $10.1177 / 0049124192021002005$.

Carvalho, R. G., \& Novo, R. F. (2014). Dimensões de personalidade e comportamentos de risco na adolescência: Um estudo com a versão portuguesa do MMPI-A. Revista Iberoamericana de Diagnóstico y Evaluación - e Avaliação Psicológica, 37(1), 203-222.

Coelho, V. A., Sousa, V., \& Figueira, A. P. C. (2014). The impact of a school-based social and emotional learning program on the selfconcept of Middle School students. Revista de Psicodidáctica, $\quad$ 19(2), 347-365. doi:10.1387/RevPsicodidact.10714

Collaborative for Academic, Social and Emotional Learning (2015). CASEL guide: Effective social and emotional learning programs middle and high school edition. Chicago, IL: Autor.

Denham, S. A., Ji, P., \& Hamre, B. (2010). Compendium of social-emotional learning and associated assessment measures. Chicago, IL: Collaborative for Academic, Social, and Emotional Learning.

Denham, S. A., Way, E., Kalb, S. C., WarrenKhot, H. K., \& Bassett, H. H. (2013). Preschoolers' social information processing and school readiness: The Challenging Situations Task. British Journal of Developmental Psychology, 31, 180-197 doi: 10.1111/j.2044-835X.2012.02085.x

Direção Geral da Saúde (2013). Portugal saúde mental em números: Programa nacional para a saúde mental. Lisboa: Direção Geral da Saúde.

Durlak, J. A., Weissberg, R. P., Dymnicki, A. B., Taylor, R. D., \& Schellinger, K. B. (2011). The impact of enhancing students' social and emotional learning: A meta-analysis of school-based universal interventions. Child Development, 82, 405-432. doi: 10.1111/j.1467-8624.2010.01564

Elliott, S. N., Frey, J. R., \& Davies, M. (2015). Systems for assessing and improving students' social skills to achieve academic competence. In J. A. Durlak, C. E. Domitrovich, R. P. Weissberg, \& T. P. Gullotta (Eds.), Handbook of social and emotional learning: Research and practice (pp. 301-319). New York, NY: The Guilford Press.

Espinosa, A. D., Menotti, I. S., \& Reyes-Lagunes, I. (2008). Validez concurrente de la escala de Deseabilidad Social de Domínguez utilizando la escala de Deseabilidad Social de MarloweCrowne. Revista Iberoamericana de Diagnóstico y Evaluación - e Avaliação Psicológica, 25(1), 125-139.

Faria, L., \& Lima Santos, N. (2005). Adaptação do Questionário de Competência Emocional (QCE) ao contexto português. Simpósio conduzido no $9^{\circ}$ Congresso Europeu de Psicologia, Granada, Espanha.

Hair, J. F., Black, W. C., Babin, B. J., Anderson, R. E., \& Tatham, R. L. (2006). Multivariate Data Analysis (6 ${ }^{\mathrm{a}}$ ed.). Upper Saddle River, NJ: Pearson Prentice Hall.

Hecht, M. L., \& Shin, Y. (2015). Culture and social and emotional competencies. In J. A. Durlak, C. E. Domitrovich, R. P. Weissberg, \& T. P. Gullotta (Eds.), Handbook of social and emotional learning: Research and practice (pp. 50-64). New York, NY: The Guilford Press.

Hu, L., \& Bentler, P.M. (1999). Cutoff criteria for fit indexes in covariance structure analysis: Conventional criteria versus new alternatives. Structural Equation Modeling: A Multidisciplinary Journal, 6(1), 1-55. doi:10.1080/10705519909540118.

LaRusso, M. D., \& Selman, R.L. (2003). The influence of school atmosphere and development on adolescents' perceptions of risks and prevention: Cynicism versus 
skepticism. In D. Romer (Ed.). Reducing adolescent risk: Toward an integrated approach. (pp. 113-122). São Francisco: Sage Publications.

LeBuffe, P.A., Shapiro, V.B., \& Naglieri, J.A. (2009). The Devereux Student Strengths Assessment (DESSA). Lewisville, NC: Kaplan Press.

Maguire, L. K., Niens, U., McCann, M., \& Connolly, P. (2015). Emotional development among early school-age children: Gender differences in the role of problem behaviors. Educational Psychology: An International Journal of Experimental Educational Psychology, 1-21. doi: 10.1080/01443410.201 5.1034090

Marôco, J. (2014). Análise estatística com o SPSS Statistics $\left(6^{\text {a }}\right.$ ed.). Pêro Pinheiro: Report Number.

Matos, M. G., Simões, C., Camacho, I., Reis, M., \& Equipa Aventura Social (2015). Relatório do estudo HBSC 2014: A saúde dos adolescentes Portugueses em tempos de recessão. Lisboa: Centro de Malária e Outras Doenças Tropicais/IHMT/UNL.

Merrell, K. (2002). School Social Behavior Scales, second edition. Eugene, OR: Assessment-Intervention Resources.

Merrell, K. W., \& Gueldner, B. A. (2010). Social and emotional learning in the classroom: Promoting mental health and academic success. Nova Iorque, NY: The Guilford Press.

Moore, K. A., \& Keyes, C. L. M. (2003). A brief history of well-being in children and adults. In M. H. Bornstein, L. Davidson, C. L. M. Keyes, \& K. A. Moore (Eds.). Well-being: Positive development across the life course (pp. 1-11). Mahwah, NJ: Lawrence Erlbaum Associates.

Nakkula, M. J., \& Nikitopoulos, C. E. (2001). Negotiation training and interpersonal development: An exploratory study on early adolescents in Argentina. Adolescence, $36(141), 1-20$.

Nunnally, J. C. (1978). Psychometric theory $\left(2^{\mathrm{a}}\right.$ ed.). Nova Iorque: McGraw-Hill.

Raimundo, R., Carapito, E., Pereira, A. I., Marques-Pinto, A., Lima, L., \& Ribeiro, T. (2012). School Social Behavior Scales (SSBS-
2): An adaptation study of the Portuguese version of the social competence scale. Spanish Journal of Psychology, 15, 14731484. doi: 10.5209/rev_SJOP.2012.v15.n3.3 9431

Raimundo, R., Marques-Pinto, A., \& Lima, M. L. (2013). The effects of a socio-emotional learning program on elementary school children: The role of pupils' characteristics. Psychology in the Schools, 50(2), 165-180. doi: 10.1002/pits.21667

Schultz, L. H., \& Selman, R. L. (2004). The development of psychosocial maturity in young children: A measure for evaluating character education programs. Journal of Research in Character Education, 2(1), 6787.

Schultz, L. H., Barr, D. J., \& Selman, R. L. (2001). The value of a developmental approach to evaluating character development programs: An outcome study of facing history and ourselves. Journal of Moral Education, 30(1), 3-27.

Schultz, L. H., Selman, R. L., \& LaRusso, M. D. (2003). The assessment of psychosocial maturity in children and adolescents: Implications for the evaluation of schoolbased character education programs. The Journal of Research in Character Education, 1(2), 67-87.

Social and Character Development Research Consortium. (2010). Efficacy of schoolwide programs to promote social and character development and reduce problem behavior in elementary school children. Washington, DC: National Center for Education Research, Institute of Education Sciences, U.S. Department of Education.

Steinberg, L. (2008). A social neuroscience perspective on adolescent risk-taking. Developmental Review, 28, 78-106. doi: 10.1016/j.dr.2007.08.002

Taksic, V. (2000). Emotional Skills and Competence Questionnaire. Rijeka: Autor.

Zins, J. E., \& Elias, M. J. (2007). Social and emotional learning: Promoting the development of all students. Journal of Educational and Psychological Consultation, 17(2-3), 233-255. doi: 10.1080/104744107014 13152 